Volume 112, Number 4

Table II. Thoracoscopic procedures and histologic findings

\begin{tabular}{lc}
\hline & No. of patients \\
\hline Procedure & \\
Biopsy of mediastinal lesions & 16 \\
Resection of mediastinal lesions & 8 \\
Lung biopsy & 5 \\
Resection of lung lesions & 5 \\
Pleural biopsy & 5 \\
Pericardial window & 2 \\
Recurrent lymphoma & 18 \\
Different diagnosis from initial lymphoma & 17 \\
Broncogenic carcinoma & 1 \\
Tuberculosis & 3 \\
Lung fibrosis & 2 \\
Interstitial pneumonitis & 3 \\
Nonspecific granuloma & 3 \\
Celomatic cyst & 1 \\
Reactive lymphnodes & 4 \\
\hline
\end{tabular}

neoplastic mass and the reactive fibrosis induced by chemotherapy and radiation therapy cause displacement and tight adhesions of mediastinal structures, and the walls of the vessels are often stiff and fragile. It is important, however, to resect and examine as much tissue as possible to rule out any residual foc of malignancy. Furthermore, these patients may have multiple lesions, and it is necessary in these cases to employ a surgical approach that makes it possible to reach them all.

From a clinical standpoint, these patients are often in poor general condition after chemotherapy and radiation therapy. If either mediastinoscopy or anterior mediastinotomy is adequate to control the lesions, it can be employed safely, ${ }^{4}$ but if a more invasive operation (such as thoracotomy or sternotomy) is required, the benefit of positive diagnosis should be carefully weighted against the increased risks of the procedure. Before the advent of
VATS, many patients could not be treated surgically because of poor general conditions and excessive risks. Our experience shows that VATS represents the solution to this problem in many cases.

Mediastinoscopy is the technique of choice for lesions located within the reach of the mediastinoscope, but this approach can be difficult and hazardous in the restaging of lymphoma because of displacement of mediastinal structures as a result of radiation therapy and chemotherapy. In addition, only small biopsy samples can be taken through this route. Anterior mediastinotomy is an excellent biopsy route, ${ }^{3,4}$ but it has limited application in a setting in which the disease may be heterogeneously distributed.

More invasive procedures such as sternal split, sternotomy, or thoracotomy, which we performed in the past, ${ }^{5}$ should be considered only when VATS is not feasible. We believe that VATS is useful in this clinical setting because severely compromised patients can also benefit from positive diagnosis and thus receive appropriate therapy.

\section{REFERENCES}

1. Filly R, Blank N, Castellino RA. Radiologic distribution of intrathoracic disease in previously untreated patients with Hodgkin's disease and non-Hodgkin lymphoma. Radiology 1976;120:277-81.

2. Mauch P, Goodman R, Hellman S. The significance of mediastinal involvement in early stage Hodgkin's disease. Cancer 1978;42:1039-45.

3. Elia S, Cecere C, Giampaglia F, Ferrante G. Mediastinoscopy versus anterior mediastinotomy in the diagnosis of mediastinal lymphoma: a randomized trial. Eur J Cardiothorac Surg 1994;57:992-5.

4. Rendina EA, Venuta F, De Giacomo T, Ciriaco P, Ricci C. Comparative merits of thoracoscopy, mediastinoscopy and anterior mediastinotomy for mediastinal biopsy. Ann Thorac Surg 1994;57:992-5.

5. Ricci C, Rendina EA, Venuta F, Pescarmona EO, Di Tolla R, Ruco LP, et al. Surgical approach to isolated mediastinal Iymphoma. J Thorac Cardiovasc Surg 1990;99:691-5.

\title{
NATIVE CARDIECTOMY IN A HETEROTOPIC HEART TRANSPLANT RECIPIENT
}

\author{
Si M. Pham, MD, Robert L. Kormos, MD, and Bartley P. Griffith, MD, Pittsburgh, Pa.
}

Heterotopic heart transplantation is traditionally indicated when the recipient has irreversible pulmonary hypertension and when the donor heart is believed to be too small to support the recipient's circulation. In long-term

From the Department of Surgery, University of Pittsburgh School of Medicine, Pittsburgh, Pa.

Received for publication Feb. 1, 1996; accepted for publication Feb. 9, 1996.

J Thorac Cardiovase Surg 1996;112:1109-11

Copyright (c) 1996 by Mosby-Year Book, Inc.

$0022-5223 / 96 \$ 5.00+0 \quad \mathbf{1 2 / 5 4 / 7 2 6 5 8}$ follow-up, mitral and tricuspid regurgitation in the native heart are commonly observed after heterotopic heart transplantation. ${ }^{1,2}$ Regurgitation of the native aortic valve is rare, however, and usually mild. We report a case of severe native aortic and mitral valve regurgitation resulting in refractory congestive heart failure in a heterotopic heart transplant recipient. The heart failure resolved after a native cardiectomy.

A patient with ischemic cardiomyopathy who had previously undergone coronary artery bypass grafting in 1970 received a heterotopic heart transplant in July 1990 at the age of 60 years. During the heart transplant operation, a piece of Dacron polyester fabric graft was used to connect 


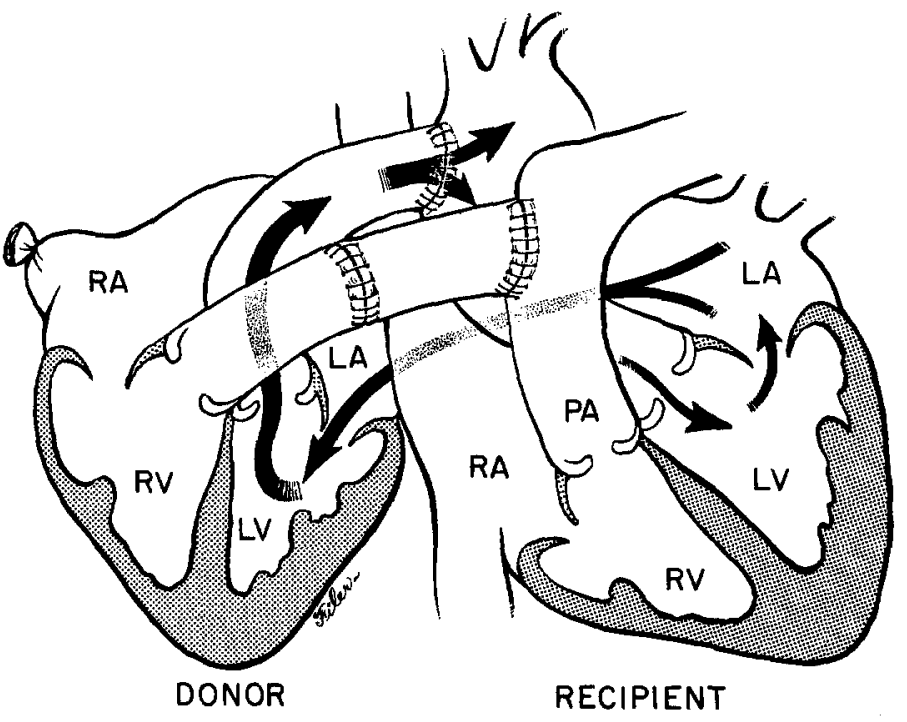

Fig. 1. Incompetence of native aortic and mitral valves allows regurgitation of blood from ascending aorta into native (recipient) left ventricle $(L V)$ and left atrium $(L A)$ and thence into donor LA. This results in decreased systemic blood flow, volume overload of transplanted LV, and congestive heart failure. Arrows indicate flow. $R A$, Right atrium; $R V$, right ventricle; $P A$, pulmonary artery.

the donor pulmonary artery to the recipient pulmonary artery. In January 1993 this pulmonary conduit became infected, necessitating replacement with fresh homograft from the descending thoracic aorta of an organ donor. In May 1993 ventricular fibrillation of the native heart developed. Cardioversion of the ventricular fibrillation was attempted, without success. The patient continued to have ventricular fibrillation for 9 months, without symptoms. In March 1994 signs and symptoms of congestive heart failure developed. Echocardiography revealed a severe regurgitation of the native aortic and mitral valves, resulting in shunting of blood from the ascending aorta to the native left ventricle and left atrium and thence to the transplanted left atrium (Fig. 1). Symptoms of congestive heart failure developed as a result of volume overloading of the transplanted heart and left-to-left shunting. In an attempt to reduce the native aortic regurgitation, which occurred more during systole than during diastole of the transplanted heart, cardioversion of the native heart was successfully performed. The native heart was maintained in sinus rhythm with quinidine sulfate. Regurgitation of the native aortic and mitral valves persisted, however, and regurgitation of native pulmonic and tricuspid valves occurred in the ensuing month. The patient's congestive heart failure symptoms became worse, changing from New York Heart Association functional class II to class III.

On May 6, 1994, the patient underwent a native cardiectomy. With cardiopulmonary bypass that used the left common femoral artery for arterial blood return and the left femoral vein and superior vena cava for venous drainage, the native heart was removed by dividing the heart around the atrioventricular groove and at the root of the pulmonary artery and aorta. Because the transplanted heart had been fixed in its original position by scar tissue, shifting or kinking of major mediastinal vessels was not observed after the native cardiectomy. The stumps of the native pulmonary artery, aorta, left atrium, and right atrium were closed (Fig. 2). Because of a $35 \mathrm{~mm} \mathrm{Hg}$ pressure gradient measured across the pulmonary arterial conduit (connecting the donor plumonary artery to the recipient pulmonary artery), enlargement of this conduit was performed with a piece of bovine pericardium. The repair reduced the gradient to $10 \mathrm{~mm} \mathrm{Hg}$ during systole of the transplanted heart.

The patient had an uneventful postoperative course. Anticoagulation therapy, which had been required after the heterotopic heart transplantation, was discontinued after the cardiectomy. At the time of this report, 21 months after his cardiectomy operation, the patient is alive and well.

This is the first reported case of congestive heart failure resulting from left-to-left shunting in a patient with a heterotopic heart transplant. The incompetent native aortic valve allows the blood to regurgitate into the native left ventricle and thence to the native left atrium (through the incompetent native mitral valve) and to the donor left atrium and ventricle (Fig. 1). This left-to-left shunting results in volume overload of the left side of the transplanted heart, decrease in forward systemic blood flood, low cardiac output, and congestive heart failure. One of the treatment options is to close the aortic valve and remove the mitral valve. This prevents regurgitation of blood into the native left ventricle and allows emptying of the left ventricle during systole of the native heart. Thrombus formation in the native left heart and the technical difficulty of closing the aortic valve without compromising the native coronary circulation are potential disadvantages of this technique. One can also remove the left ventricle, close the aortic valve and the left atrial stump, and leave the native right ventricle to help support the venous circulation. This operation was described by Losman, Curcio, and Barnard ${ }^{3}$ in 1978 in a patient who 


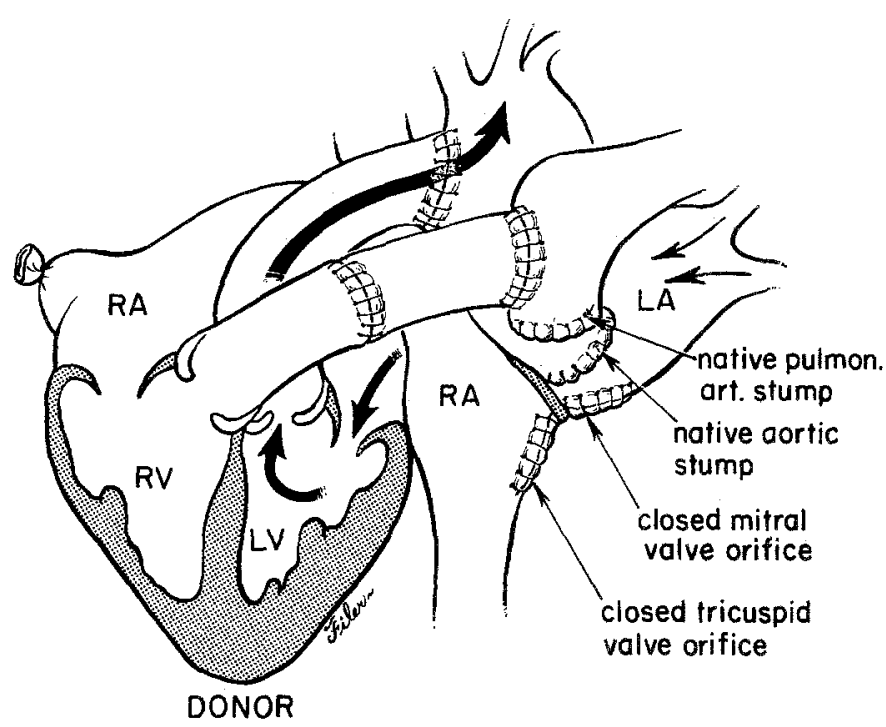

Fig. 2. Native cardiectomy at atrioventricular junction with closure of native atrial, aortic, and pulmonary arterial stumps. Arrows indicate flow. $R A$, Right atrium; $L A$, left atrium; $R V$, right ventricle; $L V$, left ventricle.

had an infected aortic valve prosthesis of the native heart. That patient survived 8 years with this "hybrid" heart. This option is suitable for those patients who still have pulmonary hypertension and therefore require a functional native right ventricle.

Another option is to perform a native cardiectomy, as was done in this case. This option is feasible only when the transplanted heart is able to function without the assistance of the native right ventricle. Our patient had demonstrated that the transplanted heart was able to support the entire circulation for a period of 9 months while the native heart was in ventricular fibrillation. Native cardiectomy is a more extensive operation than closure of the aortic valve; however, the potential problem of thrombus formation and endocarditis in the native heart is eliminated.
We thank David C. K. Cooper, MD, PhD, and Dimitri Novitzky, MD, for their valuable advice.

\section{REFERENCES}

1. Desruennes M, Muneretto C, Gandjbakhch I, Kawaguchi A, Pavie A, Bors V, et al. Heterotopic heart transplantation: current status in 1988. J Heart Transplant 1989;8:479-85.

2. Hildebrandt $A$, Reichenspurner $H$, Gordon GD, Horak AR, Odell JA, Reichart B. Heterotopic heart transplantation: mid-term hemodynamic and echocardiographic analysis--the concern of arteriovenous-valve incompetence. $J$ Heart Transplant 1990;9:675-82.

3. Losman JG, Curcio CA, Barnard CN. Normal cardiac function with a hybrid heart. Ann Thorac Surg 1978;26:177-80.

\section{CLINICAL ENDOVASCULAR PLACEMENT OF BRANCHED GRAFT FOR TYPE B AORTIC DISSECTION}

Kanji Inoue, MD, ${ }^{a}$ Mitsuru Sato, ME, ${ }^{\mathrm{b}}$ Tomoyuki Iwase, MD, ${ }^{\mathrm{c}}$ Yuki Yoshida, MT, ${ }^{\mathrm{b}}$ Terumitsu Tanaka, MD, Shunichi Tamaki, MD, ${ }^{\mathrm{c}}$ and Ario Yamazato, MD, Kyoto, Japan

The concept of a catheter-based strategy for managing aortic dissections and aneurysms continues to be exciting and promising. Most type $\mathrm{B}$ aortic dissections have initial

From the Departments of Cardiovascular Surgery, ${ }^{a}$ Clinical Laboratory, ${ }^{b}$ and Cardiology, ${ }^{\mathrm{c}}$ Takeda Hospital, Kyoto, Japan.

Received for publication Jan. 18, 1996; accepted for publication Feb. 15, 1996.

J Thorac Cardiovasc Surg 1996;112:1111-3

Copyright (c) 1996 by Mosby-Year Book, Inc.

$0022-5223 / 96 \$ 5.00+0 \quad \mathbf{1 2 / 5 4 / 7 2 8 9 7}$ intimal tears just beyond the left subclavian artery, and a straight graft with a side branch would be necessary to obliterate such a tear. We report the successful placement of a branched graft in a patient with type B aortic dissection.

A 51-year-old woman who was believed to have acute aortic dissection was referred to our hospital on May 9 , 1995. The patient's condition was managed medically because the false lumen of the descending thoracic aorta was found to be completely thrombosed by transesophageal echocardiography and contrast computed tomography. Transesophageal echocardiography during admission demonstrated that the false lumen gradually disappeared. 